\title{
Preparing the lethal hit: interplay between exo- and endocytic pathways in cytotoxic $T$ lymphocytes
}

\author{
Hsin-Fang Chang ${ }^{1} \cdot$ Hawraa Bzeih $^{1} \cdot$ Praneeth Chitirala $^{1} \cdot$ Keerthana Ravichandran $^{1} \cdot$ \\ Marwa Sleiman $^{1} \cdot$ Elmar Krause $^{1} \cdot$ Ulrike Hahn $^{1} \cdot$ Varsha Pattu $^{1} \cdot$ Jens Rettig $^{1}$ (i)
}

Received: 27 April 2016/Revised: 8 August 2016/Accepted: 29 August 2016/Published online: 1 September 2016

(C) The Author(s) 2016. This article is published with open access at Springerlink.com

\begin{abstract}
Cytotoxic T lymphocytes patrol our body in search for infected cells which they kill through the release of cytotoxic substances contained in cytotoxic granules. The fusion of cytotoxic granules occurs at a specially formed contact site, the immunological synapse, and is tightly controlled to ensure specificity. In this review, we discuss the contribution of two intracellular compartments, endosomes and cytotoxic granules, to the formation, function and disassembly of the immunological synapse. We highlight a recently proposed sequential process of fusion events at the IS upon target cell recognition. First, recycling endosomes fuse with the plasma membrane to deliver cargo required for the docking of cytotoxic granules. Second, cytotoxic granules arrive and fuse upon docking in a SNARE-dependent manner. Following fusion, membrane components of the cytotoxic granule are retrieved through endocytosis to ensure the fast, efficient serial killing of target cells that is characteristic of cytotoxic T lymphocytes.
\end{abstract}

Keywords Correlative light and electron microscopy .

Early endosomes - Late endosomes .

Recycling endosomes $\cdot$ SNARE proteins .

Total internal reflection fluorescence microscopy

\section{Abbreviations}
CG
Cytotoxic granule
CLEM
Correlative light and electron microscopy

Jens Rettig

jrettig@uks.eu

1 Cellular Neurophysiology, Center for Integrative Physiology and Molecular Medicine, Saarland University, 66421 Homburg, Germany

$\begin{array}{ll}\text { CTL } & \text { Cytotoxic T lymphocyte } \\ \text { EE } & \text { Early endosomes } \\ \text { ICAM-1 } & \text { Intercellular adhesion molecule 1 } \\ \text { IS } & \text { Immunological synapse } \\ \text { LAMP1 } & \text { Lysosomal-associated membrane protein 1 } \\ \text { LE } & \text { Late endosomes } \\ \text { LFA-1 } & \text { Lymphocyte function-associated antigen 1 } \\ \text { PtdIns(3)P } & \text { Phosphatidyl-inositol-3-phosphate } \\ \text { pMHC1 } & \text { Peptide-major histocompatibility complex } \\ & \text { type 1 } \\ \text { Rab } & \text { Ras-associated binding proteins } \\ \text { RE } & \text { Recycling endosomes } \\ \text { SMAC } & \text { Supra-molecular activation cluster } \\ \text { Sybki } & \text { Synaptobrevin2-mRFP knockin } \\ \text { Syb2 } & \text { Synaptobrevin2 } \\ \text { SNARE } & \text { Soluble N-ethylmaleimide-sensitive factor } \\ & \text { attachment receptor } \\ \text { SIM } & \text { Structured illumination microscopy } \\ \text { TCR } & \text { T cell receptor } \\ \text { TIRFM } & \text { Total internal reflection fluorescence } \\ & \text { microscopy } \\ \text { TGN } & \text { Trans-Golgi network } \\ & \end{array}$

\section{Introduction}

Cytotoxic T lymphocytes (CTL) are a central part of the cellular immune system. They recognize and kill infected cells of the body through directed release of cytotoxic substances. By their T cell receptor (TCR) they specifically recognize pMHC1 molecules on target cells carrying a cognate antigen. This highly specific interaction initiates the formation of an immunological synapse (IS) between the two cells and activates a signaling cascade that results in a series of cellular events ultimately leading to target cell 
death. The molecular trigger for IS formation involves a protein/protein interaction between the CTL adhesion molecule LFA-1 (Lymphocyte function-associated antigen 1) and the target cell membrane protein ICAM-1 (intercellular-adhesion-molecule 1). Into that region of interaction all proteins are recruited and organized in a socalled SMAC (supra-molecular activation cluster), which is essentially needed for the killing process. The organization of the SMAC involves polarization of proteins, cytoskeleton and organelles towards the IS. A microtubular network is established that transports cytotoxic granules (CG), containing cytotoxic substances such as granzymes and perforin, towards the IS. Finally, fusion of the CG with the plasma membrane occurs, thereby releasing cytotoxic substances into the cleft between CTL and target cell underneath the central SMAC. These substances diffuse to the plasma membrane of the target cell and induce apoptosis. Genetic defects leading to impaired CTL function result in life-threatening diseases like hemophagocytic lymphohistiocytosis, Griscelli syndrome 2, Chediak-Higashi syndrome and many others [1, 2], underlining the importance of this process.

All above processes are described in detail by recent reviews, which the reader may refer to [3-5]. The present review summarizes intracellular vesicle trafficking events that provide the transfer of all necessary molecular components to the IS. We also highlight the process of granule retrieval (endocytosis) after fusion and its significance to CTL function in killing multiple target cells (serial or simultaneous) [6]. A prerequisite of serial killing is the highly synchronized delivery of proteins at the IS which are needed for the fusion process as well as a synchronized endocytosis and recycling of earlier exocytosed membrane material and the generation and maturation of new CG. Since all these processes are interconnected by the endosomal network, in the first part of the review we will focus on endosomal pathways in general. In the second and third part of the review, CG maturation and recycling in CTLs will be described in particular.

\section{Endosomal network}

The endosomal network is a complicated and still not fully understood pool of intracellular compartments and vesicles. Membrane material and extracellular cargo are taken up by both clathrin-dependent and clathrin-independent endocytosis and give rise to endocytic vesicles. These vesicles fuse with early endosomes (EE) and mature into late endosomes (LE). During this process recycling endosomes bud off from EE and the remaining LE fuse with lysosomes. Moreover, newly synthesized material from the Golgi apparatus is fed into the endosomal network and vice versa (Fig. 1; [7-9]) to supply enzymes, membrane receptors and necessary membrane material to different endosomes (Fig. 1).

Over the last two decades, knowledge about the function and regulation of endosomes has substantially increased. It was found that an endosomal network exists, that not only degrades endocytosed material through fusion with lysosomes, but also recycles cargo-receptors back to the membrane via recycling endosomes. In hematopoietic cells such as CTLs, even newly generated secretory vesicles (secretory lysosomes [10]) can bud off the Golgi network and are filled with cargo such as perforin or granzymes by connections of the endosomal compartments with the Golgi apparatus [11]. In the following paragraphs, we will describe in detail the different compartments of the endosomal network. For T cell function, the endosomal network is not only interesting because of the mentioned housekeeping functions, but also for the fact that cytotoxic granules as secretory lysosomes are produced by this network. This makes a detailed understanding of the function and the components of endosomal pathways necessary for understanding immune function.

\section{Early/sorting endosomes}

The first endocytic compartment, which accepts incoming cargo internalized at the plasma membrane, is the early endosome (EE), sometimes also called the sorting endosome. It was already shown that newly formed endocytic vesicles undergo homotypic fusion or they fuse with preexisting EE in a process that requires SNARE and Rab (Ras-associated binding) proteins [12]. The most extensively investigated protein of the Rab family is Rab5. It is attributed to many different functions in early endocytic events. Rab5 can bind and activate the PtdIns3P-kinase with a primary role of generating PtdIns3P (phosphatidylinositol-3-phosphate), the most abundant phosphoinositide in the membrane of the EE [13, 14]. Another interacting partner of Rab5 is the early endosomal antigen 1 (EEA1), the most important protein related to the process of endocytic membrane docking and fusion at the EE. It is thought to be exclusively localized on the EE [15]. The dual binding of EEA1 to Rab5 and PtdIns3P tunes its localization to EE membranes [16], and its interaction with an endosomal SNARE complex makes it absolutely essential for the EE fusion in vivo [17]. Meanwhile, more proteins of the Rab family are found to be located on the EE, namely Rab10, Rab14, Rab21, and Rab22 [18].

A new approach to identify EE-specific proteins was published by Duclos and coworkers [19]. They succeeded in isolating early and late endosomes/lysosomes in macrophages and late endosomes/lysosomes in immature and mature dendritic cells and investigated the protein content 
(a)

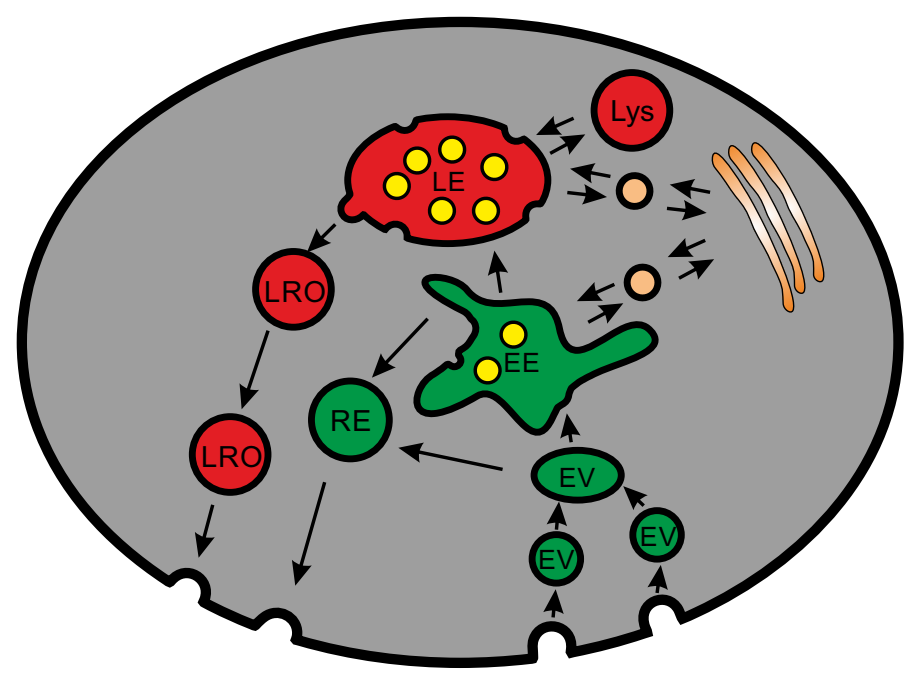

(b)



Fig. 1 Endosomal pathways. a Overview of the major endosomal pathways in mammalian cells. The plasma membrane with protein cargo is endocytosed and forms intracellular endocytic vesicles (EV). Those vesicles homotypically fuse with each other and subsequently fuse with early endosomes (EE). EE are major sorting compartments within the cell. Endocytosed material is sorted into tubular structures and bud off as recycling endosomes (RE) which migrate back to the plasma membrane and exocytose there. Moreover, a process starts in early endosomes which results in the formation of endosomal carrier vesicles (yellow). During retro- and anterograde connections to the trans-Golgi endosomal cargo, newly formed proteins are exchanged. By an ongoing production of endosomal carrier vesicles and RE, the

of these organelles by mass spectrometry. The most challenging step in this procedure was to reach a sufficiently high degree of purification of these endocytic organelles to perform reliable proteomics analysis. They found that most of the Rab proteins such as Rab11a, Rab21, Rab22a, Rab14 Rab1a and Rab5 are localized on EEs.

Depending on its respective function, a cargo protein present in an EE can be directed to three different destinations: the lysosome for degradation, the trans-Golgi network (TGN) or the cell surface via RE. The sorting of membrane proteins to multiple destinations requires an acidic luminal $\mathrm{pH}(\mathrm{pH} \sim 6.0)$ that causes the ligands to

\section{to Golgi}

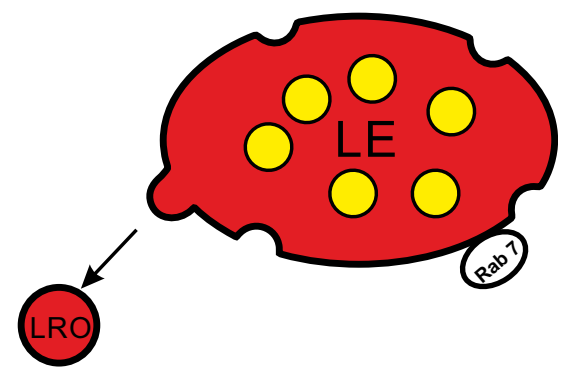

EE changes its shape and molecular composition and becomes a late endosome (LE). Finally late endosomes fuse with lysosomes (Lys) in which the remaining cargo mainly localized in endosomal carrier vesicles (multivesicular body) is degraded by hydrolytic enzymes. In some cell types such as cytotoxic T lymphocytes, LE can produce lysosomal-related organelles (LRO) that, as secretory lysosomes, become released by regulated exocytosis. Specific cargo may be inserted into LRO via a transport pathway from trans-Golgi via LE to LRO. b Closeup of EE and LE with some important molecular components; early endosomal antigen 1 (EEA1), endosomal sorting complexes required for transport (ESCRT), small GTPases (Rab5, Rab7, Rab11), sorting nexin (SNX)

dissociate from their receptors [20]. After that step a specialized machinery recognizes the cargo proteins and partitions them into discrete domains within the EE, thereby preparing the delivery to the appropriate destination. These processes are associated with morphological changes of the early endosome [21]. Electron microscopy images revealed that the EE is a dynamic compartment with a high homotypic fusion capacity and at least two functionally distinct and separate microdomains [11], namely cisternal regions or thin tubules $(\sim 60 \mathrm{~nm}$ diameter, several hundred $\mathrm{nm}$ length) and large internal vesicles ( 300-400 $\mathrm{nm}$ diameter) [22]. In general, it appears that 
cargo located after redistribution in tubules will be recycled to the plasma membrane via $\mathrm{RE}$ while the content of internal vesicles (endosomal carrier vesicles-ECV) will undergo degradation [8], preferably after the EE has matured into an LE. In case of recycling of receptors from EE back to other membranes it was shown that the formation of comprehensive endosomal tubular structures facilitates this process [12] and that actin and intact microtubules are required for both endosomal tubulation and fission [9, 23].

The transport of cargo from EE to the TGN [24] mediates the retrieval of various transmembrane receptors, such as the cation-independent mannose 6-phosphate receptor [25]. It is initialized by a retromer machinery which is preferentially recruited to maturing EEs containing increasing concentrations of PtdIns(3,5)P2 generated by PIKfyve kinase [26] and an increased number of intraluminal vesicles [27], both hallmarks of maturation into late endosomes.

The intraluminal vesicles themselves are part of the degradative function of $\mathrm{EE}$. To build up intraluminal vesicles, the cytosolic domains of endocytosed receptors become conjugated by ubiquitin on flat clathrin lattices that are present only on selected EE membranes. As a next step proteins of the "endosomal sorting complexes required for transport" (ESCRT 0-III) will bind to endosomal membranes in a sequential manner. First, ESCRT-0 binds to PtdIns3P of the EE membrane and clusters the ubiquitinylated proteins by multiple binding sites. ESCRT-I and ESCRT-II arrive and bind to the complex by interacting with each other, the cargo and the membrane. Finally, ESCRT-III binds to a subunit of ESCRT-II (VPS25) and initiates the inward vesiculation at the limiting membrane of the sorting endosome [28]. The production of intraluminal vesicles from $\mathrm{EE}$ and also LE is an ongoing process, resulting in so-called multivesicular bodies (MVB) [29]. The sorting of MHC class II molecules within the EE for example is triggered by such mechanisms [30].

As the shape of an EE changes and the number of internal vesicles increases during maturation into LE, its $\mathrm{pH}$ further decreases to $\mathrm{pH}$ values of 5.5-4.5. Moreover, it actively migrates within a cell. Early endosomes have been shown to move centripetally to the juxtanuclear position following endocytosis of cargo at the cell periphery. Attached motor proteins regulate their complex motion, the interaction with other endosomes and organelles and with the associated microtubule network [9].

\section{Recycling endosomes}

As mentioned in the previous section, many types of cargo that are endocytosed and collected in EE are sorted into tubular structures of the EE. After sorting, vesicular structures bud off from the EE and migrate as recycling endosomes (RE) towards the plasma membrane to incorporate still functional receptors and other integral membrane proteins back into the plasma membrane [9, 31, 32]. For many, if not all cell types, from a quantitative point of view, the recycling endosome pathway is the major route of vesicle trafficking [7, 33], emphasizing its importance for maintaining plasma membrane homeostasis and, therefore, cellular function.

The cargo transported via this pathway is manifold such as LDL receptors [34], MHC receptors [35], CD3 receptors [36, 37] or transferrin receptors [38]. In addition, solutes, SNARE and SNARE-associated proteins and G-protein coupled receptors are also recycled via RE. It can be assumed that almost every reusable cargo undergoes recycling via $\mathrm{RE}$, sometimes with a (currently not fully understood) switch between recycling pathways and degradation [39, 40].

Typical for recycling endosomes is the presence of Rab11 as a marker protein [41]. For fission from EE several proteins like sorting nexins (SNX) [42] and dynamin [43] are necessary. SNARE proteins like VAMP8 may drive fusion of RE at the plasma membrane [44]. The $\mathrm{pH}$ of $\mathrm{RE}$ is around 6.5 and, therefore, slightly higher as in EE, probably because of the lack of v-ATPases [45].

Aside from RE, another group of vesicles also participates in the recycling processes. These recycling vesicles constitute the so-called fast or rapid recycling [9]. They are rapidly produced after endocytosis-either shortly after fusion to EE or even before. In contrast, the classical RE is produced later after an EE has reached a position deep into the cell near the MTOC (slow recycling).

For fast recycling, Rab4 [46] and Rab35 [47] appear to be important, though their function is not entirely clear. Examples of cargo going through the fast recycling pathway might include the transferrin receptor [48] or membrane lipids [49]. Since the fast recycling vesicles do not possess Rab11 (a classical RE marker) and are not a product of classical EE sorting, they do not belong to the group of RE.

\section{Late endosomes}

There is ongoing debate about the exact step at which an EE becomes an LE, because the transition of EE to LE is a continuous process driven by fission and fusion processes which gradually change the character of endosomes [50]. Therefore, the definition is mostly operational and does not necessarily reflect a different function. For example, both subpopulations can exchange material with the Golgi and can send vesicles for exocytosis to the plasma membrane [51]. Most authors agree that an EE is characterized by two marker proteins EEA1 and Rab5 [13, 52] which are 
missing in LE. LE or related organelles are in contrast characterized by the presence of Rab7 [53]. In contrast to $\mathrm{EE}$, the $\mathrm{pH}$ in $\mathrm{LE}$ is decreased substantially to values between 5.0 and 4.5 .

A mature LE has morphologically lost its tubular structures and possesses a huge number of intraluminal vesicles. The membrane of those vesicles contains proteins that are endocytosed from the plasma membrane and intended for degradation. In contrast, most material that is intended for recycling to the PM might not be present. The fate of a mature LE is unidirectional in its progress and will eventually fuse with a lysosome and become itself a lysosome. Decisive for the fusion process is Rab7 and an effector complex which is termed HOPS in yeast [54, 55]. In mammals, it is probably composed of different SNAREs and SNARE-associated proteins [56].

\section{Lysosomes}

Lysosomes are characterized by their acidic $\mathrm{pH}(\mathrm{pH}$ 4.5-4.0) and a high concentration of proteases, nucleases and lipases. Accordingly, their function is hydrolytic degradation of all content that is delivered by late endosomes. The lysosomal-associated proteins LAMP-1 and LAMP-2, which constitute $50 \%$ of the total lysosomal membrane protein, are useful for identification [57].

A specialized subspecies of lysosomes are secretory lysosomes, which can be found in hematopoietic cells [10]. As the name implies, those lysosomes are intended to undergo regulated exocytosis. Cytotoxic granules in $\mathrm{T}$ lymphocytes belong to this group and will be described in the next section.

\section{Cytotoxic granules}

Cytotoxic granules (CG) are the primary effector organelles of CTLs. They undergo exocytosis at the CTL: target cell interface called the immunological synapse (IS) to release their cytotoxic contents such as perforin, granzymes and granulysin which all induce target cell death. CGs are believed to be hybrid organelles having properties of conventional lysosomes and secretory granules and for this reason are referred to as secretory lysosomes or lysosomalrelated organelles. This classification is made because CGspecific markers such as perforin and granzyme co-localize with conventional lysosomal markers such as LAMP-1 and LAMP-2, lysosomal transmembrane proteins such as CD63, soluble proteins such as cathepsins and other lysosomal hydrolases such as alpha-glucosidases and acid phosphatases, clearly demonstrating a lysosomal origin of CGs $[58,59]$.

\section{Composition, structure and function of CGs}

Secretory lysosomes in CTLs show a heterogeneous appearance in electron micrographs varying not only in size (300-700 nm), but also in the amount of electron-dense matrix [60]. Antibody labeling of ultrathin cryosections revealed that there are many classes of mature secretory lysosomes that might represent intermediate endosomal steps of CG maturation. Owing to this variability in the lyso-/ endosomal nature of CGs, specific marker proteins cannot be used to define the intermediate stages since they may also be located on diverse vesicles of the endosomal network. As a matter of fact, even the presence of perforin and granzymes alone, the specific effector proteins of CGs, cannot be used to define mature CGs because they are also present in other endosomal compartments such as LE and multivesicular bodies albeit in different amounts, therefore, representing different maturation stages. Despite the lack of a clear CG marker, it is believed that among the heterogenous vesicles identified in CTLs, those packed with an electron-dense proteoglycan core are most probably the most mature CGs [61]. This interpretation is strengthened by our own research, where we used the v-SNARE responsible for final CG fusion at the IS, synaptobrevin2, as a CG marker in correlative light and electron microscopy (CLEM) [60, 62]. Adding to the complexity, however, is the possibility that more than one population of mature CGs exist. Schmidt and coworkers $[63,64]$ demonstrated, by combining density gradient centrifugation, proteomics and electron microscopy that two subpopulations of CGs exist. Proteomic profiling of T cell organelles separated by density gradient centrifugation revealed the presence of two species of lysosome-related organelles: a larger, electron-light clear fraction with a diameter between 300 and $700 \mathrm{~nm}$ and a smaller, electrondense dark fraction with a diameter of less than $300 \mathrm{~nm}$. The larger fraction was enriched in FasL and classical lysosome makers such as LAMP-1, CD63 and cathepsinD, while the smaller fraction contained cytotoxic effector molecules such as granzymes, perforin and granulysin. The two populations still share $70 \%$ of approximately 400 proteins found. Schmidt and coworkers conclude that one population induces target cell membrane attack via perforin and granzymes and the second population induces target cell apoptosis via activation of target-cell Fas-receptors. This study contradicts other studies that have demonstrated co-localization of FasL with perforin/granzymes [65], adding ambiguity to the ultrastructural identity of mature CGs.

Since CGs are the effector organelles of CTLs, during infection they are transported along the microtubular network of the CTL towards the IS where a low number of CGs (1-5) fuse with the plasma membrane to release their content and kill the infected target cell [66]. Fusion is driven by several SNARE proteins from which 
synaptobrevin 2 was identified as the vesicular SNARE [62] and syntaxin11 [67] as a target SNARE on the plasma membrane. In addition to SNARE proteins, SNARE-associated proteins like Munc18-2 function as a binding partner for syntaxin11 [68]. Rab27a and Munc13-4 are the molecules required for the essential CG pre-fusion steps such as docking and priming, respectively [69-71].

\section{Maturation of CGs}

Cytotoxic granules (CGs) are formed from precursor organelles through a series of membrane transport steps (involving TGN and endosomes). The cytotoxic effector molecules perforin and granzymes are transported from the trans-Golgi network to budding endosomal vesicles that are possibly early endosomes. Granzyme A and B are translated into protein at the rough endoplasmic reticulum and mature and are targeted to the Golgi apparatus where both bind to mannose-6-phosphate receptors [72]. Luminal vesicles containing granzymes as cargo bud off from the trans-Golgi aided by the ESCRT complex (endosomal sorting complex required for transport). The luminal vesicles eventually mature by accumulation of cytotoxic effector proteins into the proteoglycan-rich dense core and mature possibly through EE and LE. Tubular EE or sorting endosomes are capable of segregating different cargo into new vesicles that bud off from the tubular endosomes and gradually mature into LE [9, 31, 32]. One might also hypothesize that the newly formed CGs along with cargo proteins are transported from EE to LE and eventually into new vesicles that bud off from LE. Since the LE bears almost all the lysosomal markers, the lysosomal origin of newly formed CGs may be obtained through LE.

de Saint Basile and coworkers proposed that the biogenesis of mature CGs is a multi-step process. This hypothesis based on work by Ménager and coworkers proposes that Munc13-4 is present on Rab11-positive recycling endosomes and Rab27a on late endosomes. The two organelles fuse to generate an intermediate precursor organelle in a Munc13-4-dependent fusion step. This hypothesis, therefore, emphasizes the function of Munc134 not only for CG exocytosis at the plasma membrane, but also for the generation of precursor intermediate exocytic vesicle [73, 74] (Fig. 2). This intermediate precursor exocytic vesicle now carrying effector molecules like Rab27a

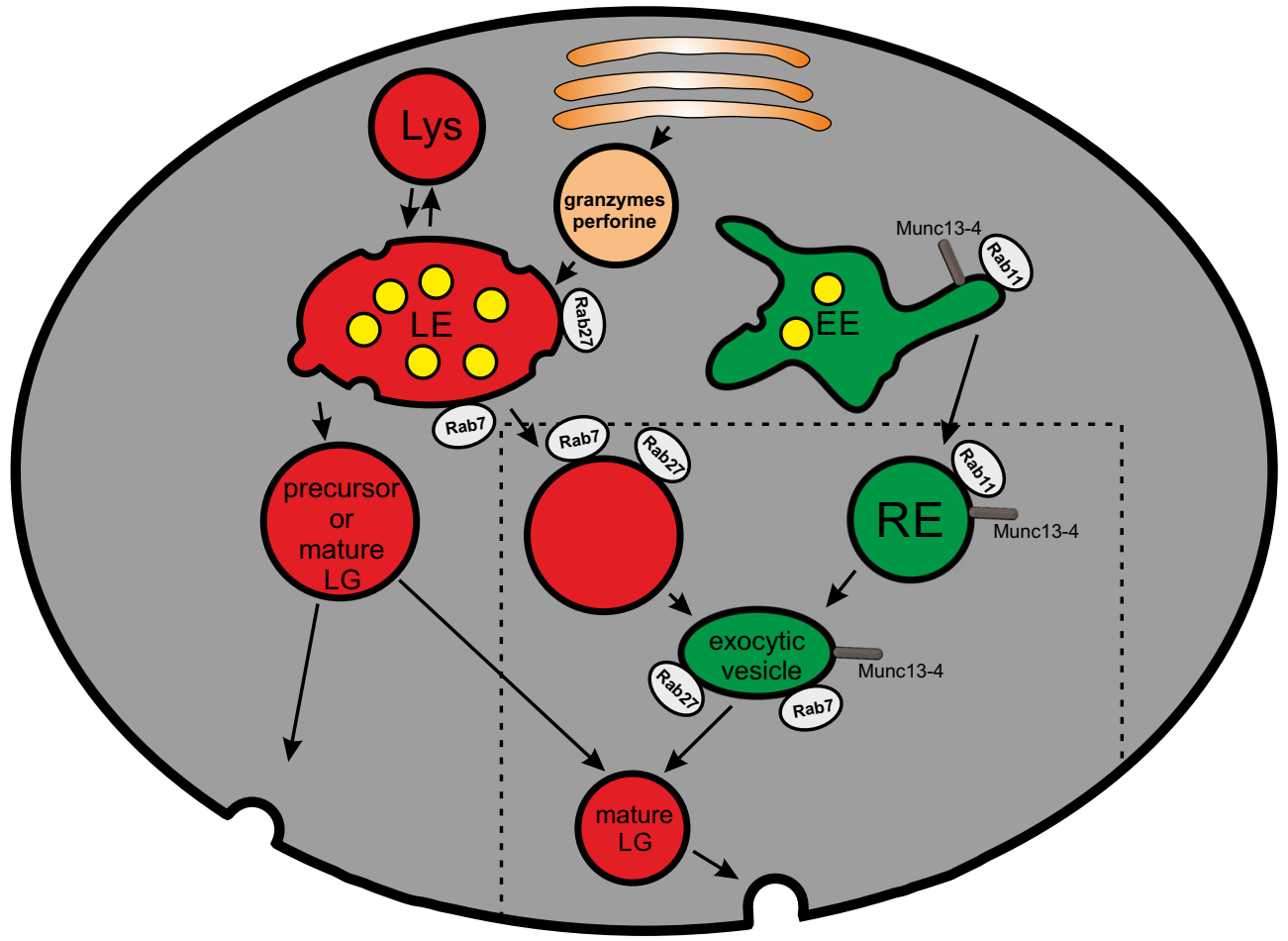

Fig. 2 Maturation of cytotoxic granules. Maturation of cytotoxic granules (CG) starts at late endosomes (LE) by budding off of secretory lysosomes (here named "precursor or mature CG"). Granzymes are incorporated into this vesicle by an anterograde transport from the Golgi network to LE and finally to CG. How other components like perforin and membrane effector proteins are incorporated is largely unknown. A new and interesting hypothesis (components inside the dashed area) emphasizes a function of recycling endosomes (RE) for maturation of
CG. Two additional fusion events were proposed. The first fusion occurs between an LE-derived vesicle carrying different Rab proteins (Rab7, Rab27) and other CG cargo and an EE-derived RE carrying Rab11 and Munc13-4. This fusion, which itself is Munc13-4 dependent, results in an intermediate named exocytic vesicle. This vesicle may then fuse with the LE-derived precursor CG to form a mature CG. Lys lysosome 
and Munc13-4 that are absolutely essential for the exocytosis of CGs, may fuse or tether with perforin/granzyme containing immature CGs to render a mature CG. The addition of other CG membrane molecules that are essential for CG fusion at the IS is also not well described.

\section{Exocytosis and endocytosis of CGs}

As outlined above, the maturation of fusogenic CGs is still very controversial. By definition, the most mature CGs must be the ones that fuse with the plasma membrane at the IS. A requirement for that fusion process must be the presence of a vesicular SNARE protein on the CG membrane. Work from our lab has shown that in murine primary CTLs, synaptobrevin2, the most important v-SNARE for neuronal synaptic vesicle exocytosis, performs this function [62]. Interestingly, the above-mentioned CLEM on CTLs derived from synaptobrevin2-knockin mice revealed two surprising pieces of data. First, the synaptobrevin2positive CGs were very homogeneous in diameter (about $350 \mathrm{~nm}$ ), indicating that, in contrast to the postulate by Schmidt and coworkers [63, 64], only one class of mature CGs exists. This finding was supported by recent combined total internal reflection fluorescence (TIRF) microscopy and membrane capacitance measurements that determined a homogeneous diameter of fusing CGs of $312 \mathrm{~nm}$ [66]. The second surprising finding of the CLEM experiment was that not all dense-core granules with a diameter of about $350 \mathrm{~nm}$ were positive for synaptobrevin2. Since synaptobrevin 2 is essentially required for the fusion of CGs with the plasma membrane, these data might imply that the synaptobrevin2-negative granules are not CGs. Whether they are precursors of mature, fusogenic CGs or belong to an entirely different class of granules remains to be elucidated. Apparently, the synaptobrevin2-knockin mouse provides an excellent tool to unravel the molecular composition of mature CGs.

The application of TIRF microscopy also enabled testing of the proposal that Rab27a/Rab11-positive endosomes fuse or tether with cytotoxic granules [73, 74] (Fig. 2). CTLs are plated on coverglass coated with anti-CD3 antibody which results in the formation of an IS at the glass/cell interface. Since the resulting evanescent wave in TIRFM extends only $150 \mathrm{~nm}$ into the CTL, labeling of granules with specific markers allows the investigation of granule mobility and fusion with high spatial (and temporal) resolution. TIRFM of CTLs in which RE were labeled with Rab11-GFP and CGs were labeled with granzymeBmCherry revealed that both vesicle types polarize to the IS and undergo fusion [67]. Importantly, though, their arrival and fusion is sequential, with RE arriving first and CGs arriving and fusing later. This sequential pattern makes sense, because Halimani and coworkers showed that syntaxin11, an essential t-SNARE for CG fusion at the IS, is transported to the IS through RE [67]. The resulting syntaxin 11 clusters in the IS plasma membrane then serve as a docking spot for arriving CGs which then form a SNARE complex to mediate fusion and release of their cytotoxic components. Further studies have verified this sequential process and identified VAMP8 as the v-SNARE mediating RE fusion at the IS [44]. Thus, RE do not fuse with CGs, and the tethering of CGs occurs through proteins like Munc13-4 and syntaxin11 that have been transported beforehand to the IS through RE (Fig. 3).
Fig. 3 Exocytosis of cytotoxic granules. Secretion of cytotoxic granules (CGs) is a sequential process requiring exocytosis of recycling endosomes (RE) as an initial step (1). Thereby REs deliver components of the exocytic machinery for CGs such as the SNARE-associated protein Munc13-4 and the SNARE protein syntaxin11 (STX11) (2). Together with further, currently unknown SNARE proteins, those components serve as a docking platform for cytotoxic granules and initiate $\mathrm{CG}$ fusion through Munc13-4-catalyzed SNARE complex formation (3)

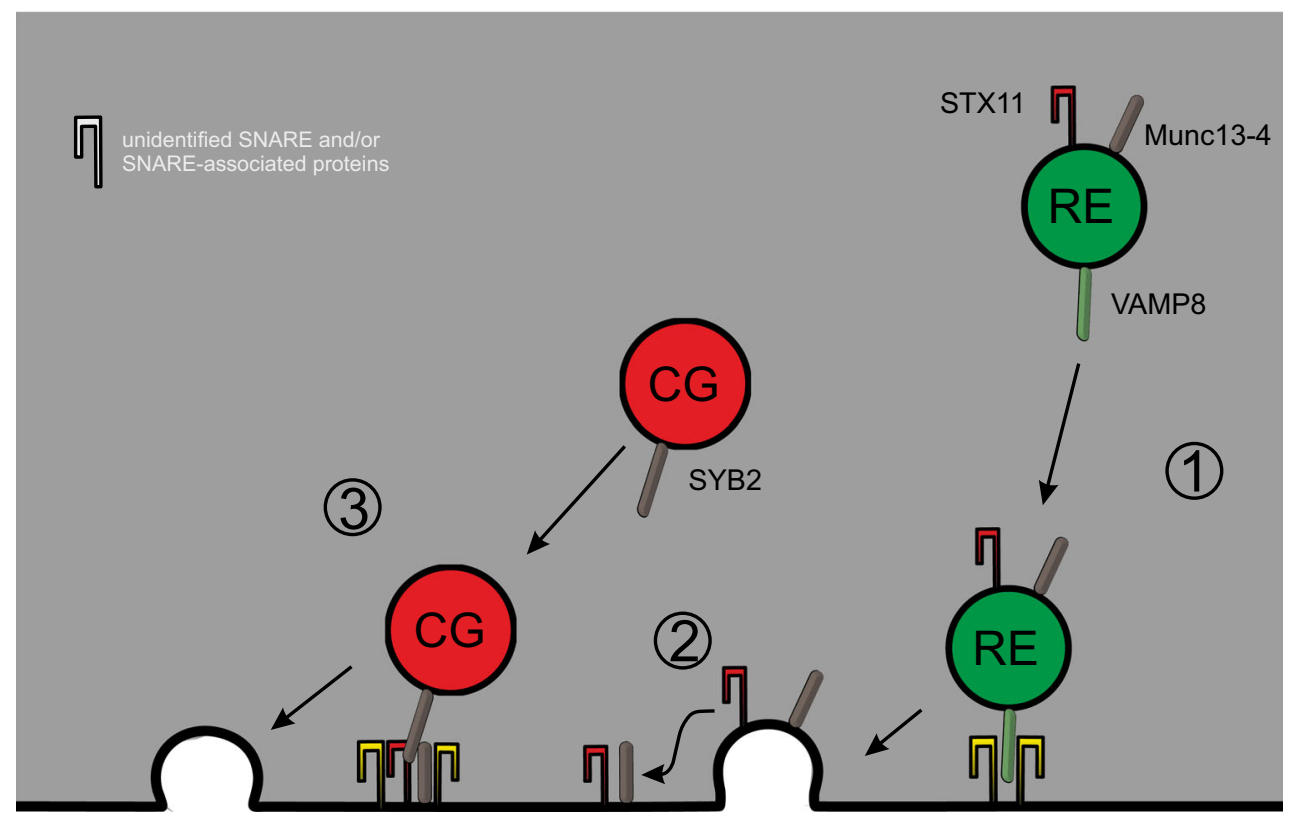


CTLs are serial killers, i.e., they can kill multiple target cells sequentially and efficiently [6, 75, 76]. Therefore, constant generation of fusogenic CGs is required. Though a constant synthesis of new CG components is theoretically possible, a much more efficient way is to retrieve used CG membrane components through endocytosis. It has been shown that essential IS membrane components like the $\mathrm{T}$ cell receptor are endocytosed through RE. Therefore, the question arises whether CG membrane components converge with IS plasma membrane components through a joint retrieval through RE. Liu and coworkers tested this hypothesis in NK cells by looking at the retrieval of LAMP-1, the major lysosomal protein that is frequently used in FACSbased degranulation assays to quantify cytotoxic granule release, under different experimental conditions in lipid bilayer-based TIRF microscopy. It was shown previously by FACS that a considerable fraction of LAMP-1 is internalized two hours after contact with target cells [77]. Liu et al. now demonstrated that both LAMP-1 exocytosis and LAMP-1 internalization occurred in a large and spatially stable cluster at the center of the IS. Both processes, as well as the correct spatial organization of receptor-ligand distribution, required the presence of the LFA-1 ligand ICAM-1 [78]. Finally, the authors showed by three-dimensional imaging of fixed cells that perforincontaining CGs were juxtaposed to the LAMP-1 internalization sites, suggesting that the IS contains an LFA-1dependent area where LG fusion and LAMP-1 internalization occur adjacent to each other. Recently, our laboratory could expand on these finding showing that while LAMP-1 internalization partially overlaps with the endocytosis of cytotoxic membrane components, synaptobrevin2 is a more specific marker protein for CG endocytosis [79]. Key molecules involved in the endocytosis of CG membrane components were dynamin, clathrin and the synaptobrevin2-specific adaptor protein CALM. Importantly, recycling of endocytosed CGs does not include RE, but rather EE and LE. Following refilling with granzyme B at the LE stage, recycled CGs are ready to fuse again and contribute about $50 \%$ to the serial killing of target cells. From these data it can be concluded that CGs are recycled, in contrast to other IS components like TCR and Munc13-4, through a specialized pathway that enters the endosomal pathway not until the EE stage.

In summary, CTLs appear to keep the endosomal pathway and the CG maturation pathway separate to fulfill their physiological function, the selective killing of target cells through controlled exocytosis of CGs at the IS. On the endocytic branch, a specialized internalization pathway for CG membrane components has been developed as well, probably to ensure a fast and efficient recycling during high killing activity.
Open Access This article is distributed under the terms of the Creative Commons Attribution 4.0 International License (http:// creativecommons.org/licenses/by/4.0/), which permits unrestricted use, distribution, and reproduction in any medium, provided you give appropriate credit to the original author(s) and the source, provide a link to the Creative Commons license, and indicate if changes were made.

\section{References}

1. Janka GE (2012) Familial and acquired hemophagocytic lymphohistiocytosis. Annu Rev Med 63:233-246

2. Meeths M, Chiang SCC, Löfstedt A, Müller ML, Tesi B, Henter JI, Bryceson YT (2014) Pathophysiology and spectrum of diseases caused by defects in lymphocyte cytotoxicity. Exp Cell Res 325:10-17

3. Angus KL, Griffiths GM (2013) Cell polarisation and the immunological synapse. Curr Opin Cell Biol 25:85-91

4. Dustin ML (2014) The immunological synapse. cancer. Immunol Res 2:1023-1033

5. Ritter AT, Angus KL, Griffiths GM (2013) The role of the cytoskeleton at the immunological synapse. Immunol Rev 256:107-117

6. Wiedemann A, Depoil D, Faroudi M, Valitutti S (2006) Cytotoxic $\mathrm{T}$ lymphocytes kill multiple targets simultaneously via spatiotemporal uncoupling of lytic and stimulatory synapses. Proc Natl Acad Sci 103:10985-10990

7. Scott CC, Vacca F, Gruenberg J (2014) Endosome maturation, transport and functions. Semin Cell Dev Biol 31:2-10

8. Kornilova ES (2014) Receptor-mediated endocytosis and cytoskeleton. Biochem 79:865-878

9. Grant BD, Donaldson JG (2009) Pathways and mechanisms of endocytic recycling. Nat Rev Mol Cell Biol 10:597-608

10. Blott EJ, Griffiths GM (2002) Secretory lysosomes. Nat Rev Mol Cell Biol 3:122-131

11. Ghosh P, Kornfeld S (2004) The GGA proteins: key players in protein sorting at the trans-Golgi network. Eur $\mathrm{J}$ Cell Biol 83:257-262

12. Maxfield FR, McGraw TE (2004) Endocytic recycling. Nat Rev Mol Cell Biol 5:121-132

13. Christoforidis S, McBride HM, Burgoyne RD, Zerial M (1999) The Rab5 effector EEA1 is a core component of endosome docking. Nature 397:621-625

14. Christoforidis S, Miaczynska M, Ashman K, Wilm M, Zhao L, Yip SZ, Waterfield MD, Backer JM, Zerial M (1999) Phosphatidylinositol-3-OH kinases are Rab5 effectors. Nat Cell Biol 1:249-252

15. Simonsen A, Lippe R, Christoforidis S, Gaullier JM, Brech A, Callaghan J, Toh BH, Murphy C, Zerial M, Stenmark H (1998) EEA1 links PI(3)K function to Rab5 regulation of endosome fusion. Nature 394:494-498

16. Lawe DC, Chawla A, Merithew E, Dumas J, Carrington W, Fogarty K, Lifshitz L, Tuft R, Lambright D, Corvera S (2002) Sequential roles for phosphatidylinositol 3-phosphate and Rab5 in tethering and fusion of early endosomes via their interaction with EEA1. J Biol Chem 277:8611-8617

17. Mills IG, Urbe S, Clague MJ (2001) Relationships between EEA1 binding partners and their role in endosome fusion. J Cell Sci 114:1959-1965

18. Jovic M, Sharma M, Rahajeng J, Caplan S (2010) The early endosome: a busy sorting station for proteins at the crossroads. Histol Histopathol 25:99-112

19. Duclos S, Clavarino G, Rousserie G, Goyette G, Boulais J, Camossetto V, Gatti E, LaBoissière S, Pierre P, Desjardins M (2011) The endosomal proteome of macrophage and dendritic cells. Proteomics 11:854-864 
20. Dautry-Varsat A, Ciechanover A, Lodish HF (1983) pH and the recycling of transferrin during receptor-mediated endocytosis. Proc Natl Acad Sci USA 80:2258-2262

21. Geuze HJ, Slot JW, Strous GJAM, Lodish HF, Schwartz AL (1983) Intracellular site of asialoglycoprotein receptor-ligand uncoupling: double-label immunoelectron microscopy during receptor-mediated endocytosis. Cell 32:277-287

22. Gruenberg J (2001) The endocytic pathway: a mosaic of domains. Nat Rev Mol Cell Biol 2:721-730

23. Marks MS, Heijnen HFG, Raposo G (2013) Lysosome-related organelles: unusual compartments become mainstream. Curr Opin Cell Biol 25:495-505

24. Bonifacino JS, Hurley JH (2008) Retromer. Curr Opin Cell Biol 20:427-436

25. Eaton S (2008) Retromer retrieves Wntless. Dev Cell 14:4-6

26. Rutherford AC, Traer C, Wassmer T, Pattni K, Bujny MV, Carlton JG, Stenmark H, Cullen PJ (2006) The mammalian phosphatidylinositol 3-phosphate 5-kinase (PIKfyve) regulates endosome-to-TGN retrograde transport. J Cell Sci 119:3944-3957

27. Arighi CN, Hartnell LM, Aguilar RC, Haft CR, Bonifacino JS (2004) Role of the mammalian retromer in sorting of the cationindependent mannose 6-phosphate receptor. J Cell Biol 165:123-133

28. Olmos Y, Carlton J (2016) The ESCRT machinery: new roles at new holes. Curr Opin Cell Biol 38:1-11

29. Williams RL, Urbé S (2007) The emerging shape of the ESCRT machinery. Nat Rev Mol Cell Biol 8:355-368

30. Luzio JP, Piper SC, Bowers K, Parkinson MDJ, Lehner PJ, Bright NA (2009) ESCRT proteins and the regulation of endocytic delivery to lysosomes. Biochem Soc Trans 37:178-180

31. Goldenring JR (2015) Recycling endosomes. Curr Opin Cell Biol 35:117-122

32. van IJzendoorn SCD (2006) Recycling endosomes. J Cell Sci 119:1679-1681

33. Steinman RM, Mellman IS, Muller WA, Cohn ZA (1983) Endocytosis and the recycling of plasma membrane. J Cell Biol 96:1-27

34. Wijers M, Kuivenhoven JA, van de Sluis B (2015) The life cycle of the low-density lipoprotein receptor: insights from cellular and in vivo studies [Miscellaneous Article]. Curr Opin Lipidol 26:82-87

35. Roche PA, Furuta K (2015) The ins and outs of MHC class IImediated antigen processing and presentation. Nat Rev Immunol 15:203-216

36. Liu H, Rhodes M, Wiest DL, Vignali DA (2000) On the dynamics of TCR:CD3 complex cell surface expression and downmodulation. Immunity 13:665-675

37. Finetti F, Paccani SR, Riparbelli MG, Giacomello E, Perinetti G, Pazour GJ, Rosenbaum JL, Baldari CT (2009) Intraflagellar transport is required for polarized recycling of the TCR/CD3 complex to the immune synapse. Nat Cell Biol 11:1332-1339

38. van Dam EM, Stoorvogel W (2002) Dynamin-dependent transferrin receptor recycling by endosome-derived clathrin-coated vesicles. Mol Biol Cell 13:169-182

39. Parachoniak CA, Luo Y, Abella JV, Keen JH, Park M (2011) GGA3 functions as a switch to promote met receptor recycling, essential for sustained ERK and cell migration. Dev Cell 20:751-763

40. Hanyaloglu AC, von Zastrow M (2007) A novel sorting sequence in the $\beta 2$-adrenergic receptor switches recycling from default to the Hrs-dependent mechanism. J Biol Chem 282:3095-3104

41. Takahashi S, Kubo K, Waguri S, Yabashi A, Shin HW, Katoh Y, Nakayama K (2012) Rab11 regulates exocytosis of recycling vesicles at the plasma membrane. J Cell Sci 125:4049-4057

42. Cullen PJ (2008) Endosomal sorting and signalling: an emerging role for sorting nexins. Nat Rev Mol Cell Biol 9:574-582
43. Tanabe K, Ohashi E, Henmi Y, Takei K (2011) Receptor sorting and actin dynamics at early endosomes. Commun Integr Biol 4:742-744

44. Marshall MR, Pattu V, Halimani M, Maier-Peuschel M, Müller M-L, Becherer U, Hong W, Hoth M, Tschernig T, Bryceson YT, Rettig J (2015) VAMP8-dependent fusion of recycling endosomes with the plasma membrane facilitates $\mathrm{T}$ lymphocyte cytotoxicity. J Cell Biol 210:135-151

45. Gagescu R, Demaurex N, Parton RG, Hunziker W, Huber LA, Gruenberg J (2000) The recycling endosome of Madin-Darby Canine kidney cells is a mildly acidic compartment rich in raft components. Mol Biol Cell 11:2775-2791

46. van der Sluijs P, Hull M, Webster P, Mâle P, Goud B, Mellman I (1992) The small GTP-binding protein rab4 controls an early sorting event on the endocytic pathway. Cell 70:729-740

47. Kouranti I, Sachse M, Arouche N, Goud B, Echard A (2006) Rab35 regulates an endocytic recycling pathway essential for the terminal steps of cytokinesis. Curr Biol 16:1719-1725

48. Harding C, Heuser J, Stahl P (1983) Receptor-mediated endocytosis of transferrin and recycling of the transferrin receptor in rat reticulocytes. J Cell Biol 97:329-339

49. Tettamanti G (2004) Ganglioside/glycosphingolipid turnover: new concepts. Glycoconj J 20:301-317

50. Stoorvogel W, Strous GJ, Geuze HJ, Oorchot V, Schwartzt AL (1991) Late endosomes derive from early endosomes by maturation. Cell 65:417-427

51. Scott CC, Gruenberg J (2011) Ion flux and the function of endosomes and lysosomes: $\mathrm{pH}$ is just the start. BioEssays 33:103-110

52. McBride HM, Rybin V, Murphy C, Giner A, Teasdale R, Zerial M (1999) Oligomeric complexes link Rab5 effectors with NSF and drive membrane fusion via interactions between EEA1 and syntaxin 13. Cell 98:377-386

53. Rink J, Ghigo E, Kalaidzidis Y, Zerial M (2005) Rab conversion as a mechanism of progression from Early to Late endosomes. Cell 122:735-749

54. Luzio JP, Gray SR, Bright NA (2010) Endosome-lysosome fusion. Biochem Soc Trans 38:1413-1416

55. Kleine Balderhaar HJ, Ungermann C (2013) CORVET and HOPS tethering complexes-coordinators of endosome and lysosome fusion. J Cell Sci 126:1307-1316

56. Pryor PR, Mullock BM, Bright NA, Lindsay MR, Gray SR, Richardson SCW, Stewart A, James DE, Piper RC, Luzio JP (2004) Combinatorial SNARE complexes with VAMP7 or VAMP8 define different late endocytic fusion events. EMBO Rep 5:590-595

57. Eskelinen E-L (2006) Roles of LAMP-1 and LAMP-2 in lysosome biogenesis and autophagy. Mol Aspects Med 27:495-502

58. Peters PJ, Borst J, Oorschot V, Fukuda M, Krähenbühl O, Tschopp J, Slot JW, Geuze HJ (1991) Cytotoxic T lymphocyte granules are secretory lysosomes, containing both perforin and granzymes. J Exp Med 173:1099-1109

59. Burkhardt JK, Hester S, Lapham CK, Argon Y (1990) The lytic granules of natural killer cells are dual-function organelles combining secretory and pre-lysosomal compartments. J Cell Biol 111:2327-2340

60. Pattu V, Halimani M, Ming M, Schirra C, Hahn U, Bzeih H, Chang H-F, Weins L, Krause E, Rettig J (2013) In the crosshairs: investigating lytic granules by high-resolution microscopy and electrophysiology. Front Immunol 4:411

61. Vollenweider I, Groscurth P (1991) Ultrastructure of cell mediated cytotoxicity. Electron Microsc Rev 4:249-267

62. Matti U, Pattu V, Halimani M, Schirra C, Krause E, Liu Y, Weins L, Chang HF, Guzman R, Olausson J, Freichel M, Schmitz F, Pasche M, Becherer U, Bruns D, Rettig J (2013) Synaptobrevin2 is the V-SNARE required for cytotoxic T-lymphocyte lytic granule fusion. Nat Commun 4:1439 
63. Schmidt H, Gelhaus C, Nebendahl M, Lettau M, Lucius R, Leippe M, Kabelitz D, Janssen O (2011) Effector granules in human T lymphocytes: proteomic evidence for two distinct species of cytotoxic effector vesicles. J Proteome Res 10:1603-1620

64. Schmidt H, Gelhaus C, Nebendahl M, Lettau M, Lucius R, Leippe M, Kabelitz D, Janssen O (2011) Effector granules in human T lymphocytes: the luminal proteome of secretory lysosomes from human T cells. Cell Commun Signal CCS 9:4

65. Bossi G, Griffiths GM (1999) Degranulation plays an essential part in regulating cell surface expression of Fas ligand in T cells and natural killer cells. Nat Med 5:90-96

66. Ming M, Schirra C, Becherer U et al (2015) Behavior and properties of mature lytic granules at the immunological synapse of human cytotoxic T lymphocytes. PLoS One. doi:10.1371/ journal.pone.0135994

67. Halimani M, Pattu V, Marshall MR, Chang HF, Matti U, Jung M, Becherer U, Krause E, Hoth M, Schwarz EC, Rettig J (2013) Syntaxin11 serves as a t-SNARE for the fusion of lytic granules in human cytotoxic T lymphocytes. Eur J Immunol 44:573-584

68. Hackmann Y, Graham SC, Ehl S, Höning S, Lehmberg K, Aricò M, Owen DJ, Griffiths GM (2013) Syntaxin binding mechanism and disease-causing mutations in Munc18-2. Proc Natl Acad Sci 110:E4482-E4491

69. Neeft M, Wieffer M, de Jong AS, Negroiu G, Metz CHG, van Loon A, Griffith J, Krijgsveld J, Wulffraat N, Koch H, Heck AJR, Brose N, Kleijmeer M, van der Sluijs P (2005) Munc13-4 is an effector of Rab27a and controls secretion of lysosomes in hematopoietic cells. Mol Biol Cell 16:731-741

70. Dudenhöffer-Pfeifer M, Schirra C, Pattu V, Halimani M, MaierPeuschel M, Marshall MR, Matti U, Becherer U, Dirks J, Jung M, Lipp P, Hoth M, Sester M, Krause E, Rettig J (2013) Different Munc13 isoforms function as priming factors in lytic granule release from murine cytotoxic T lymphocytes. Traffic Cph Den 14:798-809
71. Elstak ED, Neeft M, Nehme NT, Callebaut I, de Saint Basile G, van der Sluijs P (2012) Munc13-4*rab27 complex tethers secretory lysosomes at the plasma membrane. Commun Integr Biol 5:64-67

72. Griffiths GM, Isaaz S (1993) Granzymes A and B are targeted to the lytic granules of lymphocytes by the mannose-6-phosphate receptor. J Cell Biol 120:885-896

73. Ménager MM, Ménasché G, Romao M, Knapnougel P, Ho C-H, Garfa M, Raposo G, Feldmann J, Fischer A, de Saint Basile G (2007) Secretory cytotoxic granule maturation and exocytosis require the effector protein hMunc13-4. Nat Immunol 8:257-267

74. van der Sluijs P, Zibouche M, van Kerkhof P (2013) Late steps in secretory lysosome exocytosis in cytotoxic lymphocytes. Front Immunol 4:359

75. Bertrand F, Müller S, Roh K-H et al (2013) An initial and rapid step of lytic granule secretion precedes microtubule organizing center polarization at the cytotoxic $\mathrm{T}$ lymphocyte/target cell synapse. Proc Natl Acad Sci USA 110:6073-6078. doi:10.1073/ pnas. 1218640110

76. Choi PJ, Mitchison TJ (2013) Imaging burst kinetics and spatial coordination during serial killing by single natural killer cells. Proc Natl Acad Sci USA 110:6488-6493

77. Bryceson YT, March ME, Barber DF, Ljunggren HG, Long EO (2005) Cytolytic granule polarization and degranulation controlled by different receptors in resting NK cells. J Exp Med 202:1001-1012

78. Liu D, Bryceson YT, Meckel T, Vasiliver-Shamis G, Dustin ML, Long EO (2009) Integrin-dependent organization and bidirectional vesicular traffic at cytotoxic immune synapses. Immunity 31:99-109

79. Chang HF, Bzeih H, Schirra C, Chitirala P, Halimani M, Cordat E, Krause E, Rettig J, Pattu V (2016) Endocytoses of cytotoxic granules is essential for multiple killing of target cells by $\mathrm{T}$ lymphocytes. J Immunol 197 (in press) 